\title{
Effect of using protocols on medical care: randomised trial of three methods of taking an antenatal history
}

\author{
Richard J Lilford, Michael Kelly, A Baines, Susan Cameron, Mairead Cave, K Guthrie, \\ James Thornton
}

\begin{abstract}
Objective-To compare the effectiveness of three methods of taking an antenatal history on the quality of obstetric care.

Design-Randomised controlled trial.

Setting-Antenatal clinic of St James's University Hospital, Leeds.

Subjects-2424 women attending the hospital for the first (booking) visit.

Interventions-Histories were taken by midwives using an unstructured paper questionnaire, a structured paper questionnaire (incorporating a checklist), or an interactive computerised questionnaire (incorporating 101 clinical reminders).
\end{abstract}

Main outcome measures-The number of clinical responses to factors arising from the antenatal booking history according to method of taking the history. Actions were categorised as medical and surgical, obstetric, personal, current symptoms and treatment, related to maternal age, and related to two common actions (cervical smear testing and dental hygiene) and were weighted for clinical importance by 10 obstetricians.

Results-Overall the unstructured questionnaire generated 1063 actions, the structured questionnaire 1146 , and the computerised questionnaire 1122 . The clinical importance of these actions was lowest for the unstructured questionnaire (overall total value score $1987 v 2182$ and 2110 for the structured and computerised questionnaires respectively). The structured questionnaire was better than the computerised questionnaire in the medical and surgical (total value score $191 v 184)$, obstetric (275 v 241), and personal $(430 v 360)$ categories but inferior in the current symptoms category (179v 191).

Conclusion-Structured questionnaires (computerised or paper) provide more and better information, and their use improves clinical response to risk factors. Computerised systems offer no further advantage in antenatal clinics.

\section{Introduction}

Concern about the quality of medical care has resulted in increased emphasis on quality control by medical audit. Audit is, however, usually based on retrospective analysis of clinical activity with a view to educating professionals when substandard practice is detected. In this study we examined the effect of different systems to prevent errors of omission before they occur.

Our study was based on a clinical setting in which many specific clinical actions may be required and for which conventional audit has documented many deficiencies-first visit to an antenatal clinic. The history taken at the booking visit is the most important source of information on obstetric risk and is crucial in planning subsequent care. The quality of medical information can be improved by use of structured questionnaires, but the number of questions that might be required in an obstetric history exceeds 250 and a branching format (in which positive responses are followed up by further questions) is therefore appropriate. Such questionnaires are cumbersome on paper but amenable to computerisation.' In obstetrics and other branches of medicine the use of computerised questionnaires with a branching format improves both the quantity and quality of information when compared with existing systems ${ }^{2}$ and the computer is well received by patients. ${ }^{3}$ However, enhanced information is of little value unless it results in improved practice. An improved response to risk factors was found when a computerised history incorporating clinical reminders was used in a medical outpatient clinic, ${ }^{4}$ but protocols on paper might have been equally effective. Moreover, clinicians may simply ignore computer generated clinical suggestions. ${ }^{5}$ We therefore investigated whether structured methods in general and computerised methods in particular could improve clinicians' response rates to risk factors, a part of clinical practice in which they often fail. ${ }^{67}$

\section{Subjects and methods}

METHODS OF HISTORY TAKING

The methods compared were referred to as manual, structured, and computer. The manual system consisted of the existing form used in St James's University Hospital, Leeds, to take a history at the booking visit. In this form 29 items of information are requested, which is at the lower end of the range for the number of specific items requested in British maternity units (29 to 136). ${ }^{2}$ The structured history was a new form designed by a team of obstetricians and midwives and modified in the light of comments from the clinical department as a whole. It contained 116 items of information (representing the upper range of detail for paper based history taking systems) and a checklist of 31 items with action suggestions for the clinician.

The computer program has been described before ${ }^{13}$ and been considerably refined over the past 10 years. Briefly, the patient and midwife face the computer screen, the midwife enters the patient's answers on the keyboard, and a printout is produced for clinical interpretation and inclusion in the notes. The program contains 273 possible questions arranged in a branching format and provides up to 101 separate action suggestions (clinical reminders) printed on the output. Risk factors and action suggestions appear in a large red typeface. A list of these action suggestions has been published ${ }^{8}$ and expanded to include new information, such as screening for infection with HIV. 
$\mathrm{p}$ Value $\left(\chi^{2}\right.$ test $)$

\begin{tabular}{|c|c|c|c|c|c|}
\hline Medical category & Manual $(n=748)$ & Structured $(\mathrm{n}=739)$ & Computer $(n=736)$ & $\begin{array}{l}\text { By intended method } \\
\text { of history taking }\end{array}$ & $\begin{array}{l}\text { By actual method } \\
\text { of history taking }\end{array}$ \\
\hline Medical and surgical & 64 & 83 & 84 & $0 \cdot 2$ & $0 \cdot 1$ \\
\hline Obstetric & 97 & 119 & 103 & NS & NS \\
\hline Personal & 133 & 232 & 192 & $<0.005$ & $<0.005$ \\
\hline Current symptoms and treatment & 79 & 95 & 97 & NS & NS \\
\hline Actions related to maternal age & 89 & 107 & 96 & NS & NS \\
\hline Cervical smear test & 476 & 455 & 442 & NS & NS \\
\hline Dental hygiene & 125 & 55 & 108 & $<0.005$ & $<0.005$ \\
\hline Total & 1063 & 1146 & 1122 & $0 \cdot 1$ & 0.05 \\
\hline
\end{tabular}

$N S=$ not significant $(p>0 \cdot 2)$.

\section{PATIENTS}

Patients scheduled to attend the antenatal clinic for the first time in their current pregnancy were registered in the study and allocated to have their histories taken with one of these systems. Before each clinic a computer program randomised women to one of the three groups in blocks of three.

\section{AUDIT OF MEDICAL RESPONSES}

After delivery the case notes of all patients were analysed by two research midwives. Of 2424 patients entered, $2373(98 \%)$ had case notes available for analysis. For the first 430 assessments of the notes the research midwives were blinded to the group allocation. This was achieved by the cumbersome process of masking the history forms. The two research midwives worked independently, each reviewing all sets of notes and recording all non-routine clinical actions carried out by doctors or midwives on the basis of the history taken at the booking visit. These included actions such as instructions for follow up care (for example, testing for glucose tolerance after previous fetal macrosomia); treatment (for example, entry in a trial of fish oil or aspirin after previous pre-eclampsia); neonatal surveillance (for example, if the mother had maternal myasthenia gravis); and a request for further information (for example, pelvimetric results in a woman who had had a caesarean section).

Actions were divided into medical categories: medical and surgical, obstetric, personal, current symptoms and treatment, related to maternal age, and two common actions that would have swamped all others if not grouped separately (carrying out a cervical smear test and giving advice on dental hygiene). We also graded each of the 101 clinical actions for importance. Ten obstetricians (eight consultants and two senior registrars) working independently graded each clinical action on an integer scale of 1 to 3 , with the mean providing a quality weighting. Thus "multiple sclerosis-review regularly" was given a low mean score of $1 \cdot 2$ (presumably because there is little effective obstetric intervention) while actions prompted by a history of alloimmune thrombocytopenic purpura obtained the maximum mean score of 3. These measures of clinical importance allowed us to weight the total number of clinical actions according to their importance to give total value scores for the three history groups.

TABLE $11-N u m b e r s$ of clinical actions in manual group and in structured and computer groups combined

\begin{tabular}{lcccc}
\hline Medical category & Manual $(\mathrm{n}=748)$ & $\begin{array}{c}\text { Structured and } \\
\text { computer }(\mathrm{n}=1475)\end{array}$ & $\begin{array}{c}\text { Relative } \\
\text { frequency }\end{array}$ & $\mathrm{p}$ Value $\left(\chi^{\prime}\right.$ test) \\
\hline Medical and surgical & 64 & 167 & 1.3 & 0.06 \\
Obstetric & 97 & 222 & 1.2 & 0.2 \\
Personal & 133 & 424 & 1.6 & $<0.005$ \\
Current symptoms and treatment & 79 & 192 & 1.2 & 0.1 \\
Actions related to maternal age & 89 & 203 & $1 \cdot 15$ & $>0 \cdot 2$ \\
Cervical smear test & 476 & 897 & 0.95 & $>0 \cdot 2$ \\
Dental hygiene & 125 & 163 & 0.6 & $<0.005$ \\
\hline Total & 1063 & 2268 & 1.1 & $<0.05$ \\
\hline
\end{tabular}

\section{Results}

COMPLIANCE

Eight hundred and eight patients were entered in each group (total 2424) and notes were retrieved for 2373 (789 manual, 792 structured, and 792 computer). Forty seven $(5.5 \%)$ randomised to the computer system were booked by an alternative method because of technical failure. All analyses were done by both the intended and actual method of history taking. Randomisation produced very similar groups with respect to gravidity and maternal age, with mean gravidity 1.9 , $2.0,1.9$ for the manual, structured, and computer methods respectively and mean maternal age $26 \cdot 7$, $27 \cdot 0$, and $26 \cdot 8$ years across the three methods of history taking.

\section{AGREEMENT BETWEEN ASSESSORS}

A total of 3331 clinical actions was identified among the 2373 patients. There was no difference in identification of clinical actions between assessors according to the method of history taking (analysis of variance; $p=0.98$ ) - that is, one of the assessors did not systematically favour a particular method of history taking when compared with the other. The number of clinical responses was therefore averaged across the two assessors.

EFFECT OF BLINDING ASSESSORS TO METHOD OF HISTORY TAKING

The proportion of clinical actions recognised for each booking method did not depend on whether the assessments were made blind $(p=0 \cdot 21)$. Thus there was no evidence of systematic observer bias. We therefore pooled the blinded and unblinded results in the subsequent analysis.

\section{NUMBER OF CLINICAL RESPONSES}

The numbers of actions carried out by staff in each medical category were analysed by the $\chi^{2}$ test for each intended method of history taking (table I). The manual system generated the smallest number of clinical actions across all medical categories, with the exception of advice about dental hygiene, which had been deliberately excluded from the two structured methods. The computer history generated most clinical responses in the important category of medical and surgical history, with the structured paper method in a 
close second place, but the difference was not significant across all three methods $(p=0 \cdot 2)$. In all other categories the structured system came out slightly ahead. Analysis by the actual (rather than intended) method of history taking slightly favoured the computer but made no substantive difference to the conclusions (table I).

We also tested the hypothesis that structured methods, computerised or manual, produce more clinical actions than do unstructured methods. Thus the mean number of actions per patient was compared between the unstructured manual method and the two structured methods combined (table II). The overall result was significant $(p<0.05)$ and $p=0.06$ for the medical and surgical category.

IMPORTANCE OF CLINICAL RESPONSES

The mean importance weightings of the various clinical actions, as judged by 10 obstetricians, were $2.24,2.32,1.87,1.93,1.62,1.90$, and 1.30 for the respective medical categories medical and surgical, obstetric, personal, current symptoms and treatment, actions related to maternal age, cervical smear testing, and dental hygiene. Thus the obstetricians attributed the greatest importance to clinical actions arising in the medical and surgical and obstetric categories of the booking history. Our omission of dental issues from the two new structured methods seems to be justified, although the presence of dental hygiene in the manual history, which otherwise produced less information than its rivals, reduced the overall differences observed.

There were no significant differences between the methods of history taking in the mean importance of the actions in the various medical categories (one way analysis of variance). The result was not significant $(p=0.06)$ when data were combined over all medical categories, the mean value scores being $1.90,1.88$, and 1.86 for the structured, computer, and manual systems respectively.

The total value scores, in which the number of items are weights for importance, were lowest for the manual system in all categories (again with the exception of dental hygiene). These differences were significant by the $\chi^{2}$ test, both overall and in certain medical categories (table III). The computer was superior to the structured history with respect to current symptoms and treatment whereas the structured history gave best results in the medical and surgical, obstetric, and personal categories.

Again, these findings were essentially unchanged when the results were analysed according to the actual as opposed to intended method of history taking. Although the computer was slightly favoured, the results were statistically similar (table III).

\section{Discussion}

Our study shows that structured methods, whether computerised or paper based, result in improvements in clinical responses. This was found in all the important sections of the history, and the differences between methods were greatest when the number of clinical responses were weighted by their importance.

McDonald found that computer prompts improved clinical practice in a hypertension clinic, ${ }^{4}$ but he did not compare the computer system against paper checklists such as those incorporated in the structured obstetric history in our study. Audit of clinical standards has shown that paper checklists are successful in antenatal care. ${ }^{9}$ The antenatal history is a particularly good model for evaluating computer histories because it is based on a well defined dataset that can be presented to the user in a predictable and logical order. Computers are most successful when used to obtain such self contained histories ${ }^{10}$ but are less able to encapsulate the patient's problem in less stereotyped encounters, such as those in a general medical ${ }^{11}$ or gynaecology ${ }^{12}$ department, where the computer is called on to emulate a partially intuitive process. ${ }^{13}$ However, we found no further improvements when the computer was substituted for detailed case notes and checklists. As antenatal care seems to be particularly suitable for computerisation, even more disappointing results might be expected from history taking systems in many other branches of medicine. Computer history systems are more expensive than structured manual systems in terms of hardware, software, and staff time, ${ }^{3}$ and we cannot recommend their use unless their performance is better than optimal paper systems.

Nevertheless, errors of omission still occur with structured histories and checklists. Clinical care might be improved by extended computer systems designed not only to capture all relevant data but also to check, later in pregnancy, that recommended clinical actions have been carried out. Computer systems have been developed to accept online data at different stages of antenatal care and the most comprehensive of these dispense with the paper record altogether. ${ }^{14}$ It should be possible to extend such systems to ensure that necessary clinical responses have been carried out-for example, extended computer systems could alert staff if a glucose tolerance test has not been ordered at the appropriate stage of pregnancy in a woman who has previously had a large baby. However, systems capable of implementing strict protocols on the basis of the logical integration of information obtained from different sources (including the community) and at different times are expensive. There is no uniformity in computer hardware and software or medical care programmes between medical care providers. Thus each system must be customised to reflect not only different computer systems but also different patterns of medical care. Before committing resources on a large scale across the world, a few selected systems should be carefully evaluated. These systems should be piloted in well circumscribed areas of medical practice, such as antenatal or intensive care, in which a finite number of widely accepted protocols can be identified. Some, but certainly not all, aspects of medical care might be sufficiently improved to warrant the cost of such integrated systems. If this is so, then comprehensive,

TABLE III-Total value scores of information obtained by three methods of history taking

\begin{tabular}{|c|c|c|c|c|c|}
\hline \multirow[b]{2}{*}{ Medical category } & \multirow[b]{2}{*}{ Manual $(n=748)$} & \multirow[b]{2}{*}{ Structured $(n=739)$} & \multirow[b]{2}{*}{ Computer $(n=736)$} & \multicolumn{2}{|c|}{$\mathrm{p}$ Value } \\
\hline & & & & $\begin{array}{l}\text { By intended method } \\
\text { of history taking }\end{array}$ & $\begin{array}{l}\text { By actual method } \\
\text { of history taking }\end{array}$ \\
\hline Medical and surgical & $142 \cdot 7$ & $190 \cdot 7$ & $184 \cdot 4$ & 0.02 & $<0.005$ \\
\hline Obstetric & $225 \cdot 5$ & $275 \cdot 2$ & $240 \cdot 8$ & $0 \cdot 15$ & 0.075 \\
\hline Personal & $250 \cdot 7$ & $430 \cdot 0$ & $360 \cdot 1$ & $<0.005$ & $<0.005$ \\
\hline Current symptoms and treatment & $152 \cdot 2$ & $179 \cdot 3$ & $191 \cdot 3$ & $0 \cdot 15$ & 0.05 \\
\hline Actions related to maternal age & $150 \cdot 0$ & $170 \cdot 4$ & $152 \cdot 7$ & $>0 \cdot 4$ & $>0 \cdot 3$ \\
\hline Cervical smear test & $904 \cdot 4$ & $864 \cdot 5$ & $839 \cdot 8$ & $>0.4$ & $>0.3$ \\
\hline Dental hygiene & 162.5 & 71.5 & $140 \cdot 4$ & $<0.005$ & $<0.005$ \\
\hline Total & $1987 \cdot 6$ & $2181 \cdot 5$ & $2109 \cdot 5$ & 0.007 & $<0.005$ \\
\hline
\end{tabular}


interactive clinical computer systems, such as that piloted by Weed more than 20 years ago, ${ }^{15}$ may yet come of age.

This work was supported by the Medical Research Council. We thank the midwives in the booking clinic for their cooperation and the consultant obstetricians at St James's University Hospital for allowing us to study women who had been referred to them. We particularly thank Mrs Margaret Barker for her invaluable clerical support, especially in masking the history forms of the first 430 subjects.

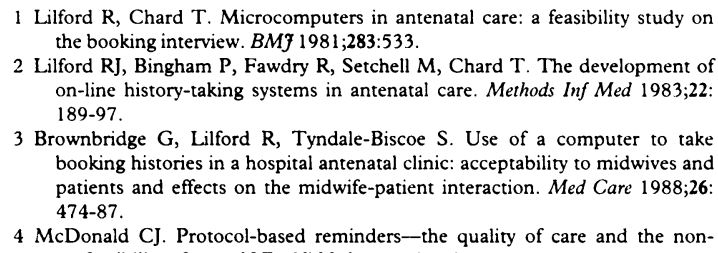
on-line history-taking systems in antenatal care. Methods Inf Med 1983;22: 189-97.

3 Brownbridge G, Lilford R, Tyndale-Biscoe S. Use of a computer to tak booking histories in a hospital antenatal clinic: acceptability to midwives and patients and effects on the midwife-patient interaction. Med Care 1988;26: 474-87.

$4 \mathrm{McDonald}$ CJ. Protocol-based reminders-the quality of care and the nonperfectibility of man. N Engl I Med 1976;195:1351-5.
5 Lilford RJ, Chard T, Bingham P, Carrigan E. Use of a microcomputer network for history taking in a prenatal clinic. Am 7 Perinatol 1985;2:143-7.

6 Guthrie KA, Songane FF, Mackenzie F, Lilford RJ. Audit of medica response to antenatal booking history. Br $\mathcal{Y}$ Obstet Gynaecol 1989:96:552-6.

7 Chng PK, Hall MH, MacGillivray I. An audit of antenatal care: the value of the first antenatal visit. $B M 7$ 1980;281:1 184-6.

8 Lilford RJ, Chard TC. The use of a small computer to provide action suggestions in the booking clinic. Acta Obstetrica et Gynaecologica faponica 1984;36:119-25.

9 Staines C. Moving forward in antenatal care-the Sighthill project, Edinburgh. Midwives Chronicle 1983;96:6-8.

10 Lilford RJ, Guthrie K; Kelly M. History-taking by computer. Bailliere's Clin Obstet Gynaecol 1990;4:723-42

11 Quaak MJ, Westerman RF, van Bemmel JH. Comparisons between written and computerised histories. BMF 1987;295:184-95.

12 Bingham P, Lilford RJ, Chard T. Strengths and weaknesses of direct patien interviewing by a microcomputer system in specialist gynaecological practice. Eur 7 Obstet Gynaecol Reprod Biol 1984;18:43-56.

13 Dreyfus HL, Dreyfus SE, Athenafion T. Mind over machine (the power of human intuition and expertise in the era of the computer). Oxford: Blackwell, 1986.

14 Gonzalez FA, Fox HE. The development and implementation of a computerised on-line obstetric record. Br f Obstet Gynaecol 1989;96:1323-7.

15 Weed LL. Medical records, medical education and patient care; the problem orientated record as a basic tool. Cleveland, Ohio: Case Western Reserve University Press, 1969

(Accepted 3 September 1992)

\title{
Audit of compliance with antenatal protocols
}

\author{
A F E Yoong, J Lim, C N Hudson, T Chard
}

\section{Abstract}

Objective-To assess the implementation of action protocols dictated by antenatal risk factors noted at the initial (booking) antenatal visit.

Design-Retrospective study of 2000 women delivered between 1 March 1990 and 29 March 1991.

Setting-Maternity department of a district general hospital supporting a multiethnic population in inner London.

Main outcome measures-Comparison of clinical actions performed against those dictated by the department's protocols. Analysis according to clinical importance, gestation at booking, maternal age, parity, birth order, ethnic origin, and certainty of gestational age.

Results-Interobserver agreement between the two auditors was good ( $x$ statistic for risk factors detected, 0.78 ; for actions generated, $0 \cdot 80$ ). Of the 15658 actions dictated by department protocols, $3673(23 \cdot 5 \%)$ were actually performed by the clinicians. The 63 combinations of risk factors and actions believed by consultants to be of particular clinical importance had an action rate of $28.3 \%$ compared with $18.6 \%$ for those considered less important $(p<0 \cdot 001)$. Mothers who first visited the hospital antenatal clinic at or before 24 weeks' gestation had $25 \cdot 2 \%$ of relevant protocols fulfilled $(\mathbf{p}<0 \cdot 001)$. Compliance was significantly improved in women aged 36 or over $(32.4 \%)$, black women $(24 \cdot 9 \%)$, and cases of uncertain gestation $(24 \cdot 5 \%)$. Parity and birth order were not associated with an altered action rate. Ethnic origin deemed as "other" (than white, black, Asian, or oriental) or "unknown" was associated with poor compliance $(19 \cdot 3 \%)$.

Conclusions-Compliance to a set of agreed protocols was poor even though a computer system was available and a protocol manual had been distributed. Protocols were more likely to be implemented in women who booked early and in some groups of women deemed at high risk including older mothers, black women, and those denoted as having uncertain gestational age.

\section{Introduction}

The process of antenatal care and the management of pregnancy is determined largely by the detection of risk factors at the first antenatal attendance (booking visit). The Sighthill scheme promoted a high standard of antenatal care by ensuring that each pregnant woman was formally assessed: features of the pregnancy were noted against a checklist of risk factors; a continuing plan of management using specialist and community services, based on locally agreed protocols, would then be organised and instituted with the help of risk cards.' A similar programme, developed at the Homerton Hospital, used a computer system to help detect obstetric risk factors. ${ }^{2}$ About half of the pregnant women attending the hospital have their booking history collected with this clinical information system; the computer generates a printed antenatal report, in which a list of relevant risk factors appears on the first page. In 1986 a detailed set of protocols for antenatal care was devised. These protocols defined 46 risk factors that could be identified at the booking visit, together with appropriate actions to be followed should a risk factor be found.

Two previous studies that analysed medical management according to recorded antenatal information concentrated on the recognition of risk factors at the booking visit rather than the adequacy of the medical response. ${ }^{34}$ In neither study were protocols for specific actions in response to specific risk factors stated to be clearly established before antenatal care was given to the mothers. We evaluated how actions based on the protocol were carried out when risk factors had been identified in mothers booked through the computer system in the maternity department of Homerton Hospital.

\section{Method}

The case notes of 2000 mothers delivered between 1 March 1990 and 29 March 1991 who had been booked on to the computer system at the Homerton Hospital were scrutinised after delivery by one of the authors (AY). The details examined included the booking history and examination and consequent antenatal care. Risk factors were scored as being present or absent or as missing information. Each of 38 possible clinical actions that might be carried out on the basis of risk factors identified at booking were recorded as having been performed or not. Risk factors were said to have been detected if printed on the 